\section{Connection Aspects of the Non-linear Schrödinger Equation}

\section{A. Grauel}

Arbeitsgruppe Theoretische Physik, Universität Paderborn, Federal Republic of Germany

Z. Naturforsch. 36a, 1118-1119 (1981); received August 24, 1981

Some geometrical features of the non-linear Schrödinger equation are studied and it is described how the Schrödinger equation can be obtained from the non-linear scattering equations. The $\operatorname{SL}(2, \mathbb{R})$-valued elements of the matrix of the non-linear scattering problem are interpreted as matrix-valued forms. We calculate the curvature form with respect to a basis of the Lie algebra. If the curvature form equals zero then we obtain the non-linear Schrödinger equation.

Wahlquist and Estabrook have given a geometric approach [1] and they find a prolongation structure for non-linear partial differential equations in two independent variables. They have applied their method to the non-l near Schrödinger equation and they discussed the relationship between the pseudopotentials and the inverse scattering method (ISM) of Zakharov and Shabat [2], and Ablowitz, Kaup, Newell and Segur [3]. Sasaki [4] has shown that the ISM is given by a completely integrable pfaffian system and that the equations of Ablowitz, Kaup, Newell and Segur can be described by a pseudospherical surface. Furthermore, Sasaki [5] has given pseudopotentials for equations of Ablowitz, Kaup, Newell und Segur.

We give a further example of the Lie algebraic method to study the geometrical aspects of the non-linear Schrödinger equation. First we cite the scattering equations (ISM) and some formulae [6]. We use the (ISM) in the form

$$
\frac{\partial}{\partial x}\left(\begin{array}{l}
\varphi^{1} \\
\varphi^{2}
\end{array}\right)=\left(\begin{array}{cc}
\eta & q(x, t) \\
r(x, t) & -\eta
\end{array}\right)\left(\begin{array}{l}
\varphi^{1} \\
\varphi^{2}
\end{array}\right)
$$

where the time evolution of the functions $\varphi^{1}(x, t)$ and $\varphi^{2}(x, t)$ are given by

$$
\frac{\partial}{\partial t}\left(\begin{array}{l}
\varphi^{1} \\
\varphi^{2}
\end{array}\right)=\left(\begin{array}{rr}
A(x, t ; \eta) & B(x, t ; \eta) \\
C(s, t ; \eta) & -A(x, t ; \eta)
\end{array}\right)\left(\begin{array}{l}
\varphi^{1} \\
\varphi^{2}
\end{array}\right) .
$$

The coefficients $A, B$ and $C$ are functions of $x$ and time $t$. The quantity $\eta$ is the eigenvalue of the

Reprint requests to Dr. A. Grauel, Arbeitsgruppe Theoretische Physik, Universität Paderborn, Warburger Str. 100, 4790 Paderborn, Fed. Rep. of Germany. scattering problem and we assume $\eta \neq \eta(t)$. The system (1) and (2) can be rewritten in matrix notation. We have

$$
\frac{\partial \varphi^{k}}{\partial x^{j}}+\sum_{p=1}^{2} \Omega_{p j}^{k} \cdot \varphi^{p}=0,
$$

where $j, k=1,2$ and $x^{1}=x, x^{2}=t$. The $\varphi^{k}(x, t)$ are the components of a two-component field on the principal bundle $P(M, G)$. The $\Omega_{p j}^{k}$ are given by the components of the matrix in (1) and (2). The theory of Cartan-Ehresmann connections (see [7]) generalizes the Gaussian curvature of a Riemannian 2-dimensional manifold. The curvature form is given by the exterior covariant derivative of the 1 -form $\omega$ on $P(M, G)$ with the values in a finite-dimensional vector space $V$ in the form

$$
\Theta=\nabla \omega=\mathrm{d} \omega \circ h,
$$

where $\Theta$ is a $\boldsymbol{g}$-valued 2 -form. The $(p+1)$-form $\nabla \omega$ is given by

$$
\begin{aligned}
& \nabla \omega\left(X_{1}, \ldots, X_{p+1}\right) \\
& =\mathrm{d} \omega\left(h X_{1}, \ldots, h X_{p+1}\right),
\end{aligned}
$$

and $h: T_{p}(P(M, G)) \rightarrow S_{p}$ the projection of $T_{p}$ (tangential space) onto its horizontal subspace $S_{p}$. The space of vertical vectors $V_{p}=T_{p} \Theta S_{p}$ lies tangential to the fibre.

In a previous paper [6] we have expressed the curvature form $\Theta$ on bundles $P(M, G)$ in the form

$$
\begin{aligned}
\Theta= & \sum_{i=1}^{3} \mathrm{~d} \omega^{i} \otimes X_{i} \\
& +\frac{1}{2} \sum_{i, j=1}^{3}\left(\omega^{i} \wedge \omega^{j}\right) \otimes\left[X_{i}, X_{j}\right],
\end{aligned}
$$

where $\omega^{k}(k=1,2,3)$ are arbitrary one-forms and $\left[X_{p}, X_{q}\right]$ is the commutator of the quantities $X_{k}$. The two-form $\Theta$ is called the curvature of the connection. The $\left\{X_{k}\right\}_{k=1}^{3}$ are a basis of the Lie algebra $\boldsymbol{g}=\mathrm{SL}(2, \mathbb{R})$, and we choose

$$
\begin{array}{ll}
X_{1} & =\left(\begin{array}{rr}
1 & 0 \\
0 & -1
\end{array}\right), \quad X_{2}=\left(\begin{array}{ll}
0 & 1 \\
0 & 0
\end{array}\right), \\
X_{3} & =\left(\begin{array}{ll}
0 & 0 \\
1 & 0
\end{array}\right) .
\end{array}
$$

In view of (3) we can write for the 1 -forms

$$
\begin{aligned}
& \omega^{1}=-(\eta \mathrm{d} x+A \mathrm{~d} t), \\
& \omega^{2}=-(q \mathrm{~d} x+B \mathrm{~d} t), \\
& \omega^{3}=-(r \mathrm{~d} x+C \mathrm{~d} t) .
\end{aligned}
$$

0340-4811 / 81/1000-1118 $\$ 01.00 / 0$. - Please order a reprint rather than making your own copy. 
If we rewrite the curvature form (6) with (7) and (8) then we obtain the expression

$$
\begin{aligned}
\Theta= & \left(q C-r B-A_{x}\right) \cdot \mathrm{d} x \wedge \mathrm{d} t \otimes X_{1} \\
+ & \left(2 \eta B-2 q A+q_{t}-B_{x}\right) \\
& \cdot \mathrm{d} x \wedge \mathrm{d} t \otimes X_{2} \\
+ & \left(-2 \eta C+2 r A+r_{t}-C_{x}\right) \\
& \cdot \mathrm{d} x \wedge \mathrm{d} t \otimes X_{3} .
\end{aligned}
$$

If we choose

$$
\begin{aligned}
& r=-q^{*}=-u^{*}, \quad A=2 i \eta^{2}+i|u|^{2} \\
& B=i u_{x}+2 i \eta u, \quad C=i u_{x}^{*}-2 i \eta u^{*},(10)
\end{aligned}
$$

then we obtain

$$
\begin{aligned}
\Theta= & \left(-2 u i|u|^{2}+u_{t}-i u_{x x}\right) \mathrm{d} x \wedge \mathrm{d} t \otimes X_{2} \\
& +\left(-2 u * i|u|^{2}-u_{t}^{*}-i u_{x x}^{*}\right) \\
& \cdot \mathrm{d} x \wedge \mathrm{d} t \otimes X_{3} .
\end{aligned}
$$

If $\Theta=0$ we obtain

$$
\begin{aligned}
i u_{t}+u_{x x}+2|u|^{2} u & =0, \\
-i u_{t}^{*}+u_{x x}^{*}+2|u|^{2} u^{*} & =0,
\end{aligned}
$$

[1] H. D. Wahlquist and F. B. Estabrook, J. Math. Phys. $16,1(1975)$ and 17, 1293 (1976).

[2] V. E. Zakharov and A. B. Shabat, Sov. Phys. JETP 34, 62 (1972).

[3] M. J. Ablowitz, D. J. Kaup, A. C. Newell, and H. Segur, Phys. Rev. Lett. 31, 125 (1973).

[4] R. Sasaki, Phys. Lett. 71 A, 390 (1979).

[5] R. Sasaki, Phys. Lett. 73 A, 77 (1979).

[6] A. Grauel, Z. Naturforsch. 36 a, 417 (1981). for the real and imaginary part of the non-linear Schrödinger equation. Moreover, from the condition $\Theta=0$ we conclude that

i) $\omega$ satisfies the Maurer-Cartan structural equation

$$
\mathrm{d} \omega+\frac{1}{2}[\omega, \omega]=0,
$$

ii) the connection in $P(M, G)$ is flat.

To summarize: We have used the Lie algebraic method to give a geometrical interpretation of the non-linear Schrödinger equation. We see that $\omega$ satisfies the structure equation of Maurer-Cartan, which implies that the canonical flat connection has zero curvature. Therefore we can say that the non-linear Schrödinger equation can be deduced from the fact that the $\operatorname{SL}(2, \mathbb{R})$ connection associated with the scattering equation has zero curvature. The Bäcklund transformation for the nonlinear Schrödinger equation is given by Lamb [8] in a different way by using the method of pseudopotentials [1].

[7] C. von Westenholz, Differential Forms in Mathematical Physics, North-Holland, Amsterdam 1978.

[8] T. P. Branson, Journal of Differential Geometry (1981).

[9] S. Kobayashi and K. Nomizu, Foundations of Differential Geometry, Vol. I, Interscience Publishers, London 1963.

[10] G. L. Lamb, Phys. Lett. 48 A, 73 (1974). 